\title{
Nanochannel Materials for Quantum Solar Energy Conversion Devices
}

\author{
Dominik Brühwilerª, Le-Quyenh Dieuª ${ }^{\mathrm{a}}$ and Gion Calzaferrib
}

\begin{abstract}
The supramolecular organization of dye molecules in one-dimensional zeolite channel systems leads to materials with intriguing properties that can be exploited for a variety of applications. Two concepts are presented outlining how zeolite-based hybrid host-guest compounds can be employed in solar energy conversion devices. The first concept describes light-harvesting and sensitization, while the second concept focuses on luminescent solar concentrators.
\end{abstract}

Keywords: Energy transfer · Host-guest · Luminescence · Solar energy conversion · Zeolite

\section{Introduction}

The concept of organizing guest molecules in robust silicate-based porous solids is well established. ${ }^{[1]}$ In this context, zeolites (pore diameter below $2 \mathrm{~nm}$ ) are popular host materials, as their crystalline frameworks provide defined channel architectures ${ }^{[2]}$ while being transparent from UV to near-infrared. One-dimensional channel systems are of particular interest, because the inclusion of chromophores can result in materials featuring optical anisotropy. ${ }^{[3]}$ The molecularsized channels offer possibilities to obtain high local concentrations of monomeric dyes. In such systems, electronic excitation energy is efficiently transported along the channels by means of Förster resonance energy transfer (FRET). ${ }^{[4]}$ Furthermore, dyes in the channels are protected by the inert aluminosilicate framework, often leading to increased stability. The interplay of confinement and enhanced guest stability has been studied for specific cases. ${ }^{[5]}$ Generally, it can be inferred that the penetration

${ }^{*}$ Correspondence: Dr. D. Brühwiler

Tel.: +41446354630

Fax: +41446356802

E-Mail: bruehwi@aci.uzh.ch

anstitute of Inorganic Chemistry

University of Zürich

Winterthurerstrasse 190

$\mathrm{CH}-8057$ Zürich

bDepartment of Chemistry and Biochemistry

University of Bern

Freiestrasse 3

$\mathrm{CH}-3012$ Bern and diffusion of reactants is limited due to the steric constraints imposed by the guestfilled channels. In the following, we present two concepts outlining the potential use of dye-nanochannel inclusion compounds in quantum solar energy conversion devices.

\section{Sensitization of Organic Solar Cells}

Fig. 1 illustrates the design of a nanochannel-based photonic antenna currently being investigated in our lab. Various zeolites featuring one-dimensional channel systems can in principle be used as host materials in such an assembly. Even mesoporous solids are interesting candidates, given the recent progress in the synthesis of ordered thin films with perpendicular orientation of the channels. ${ }^{[6]}$ For guest molecules smaller than $7 \AA$ (at least in two dimensions), zeolite $\mathrm{L}$ is an ideal choice. Crystals of zeolite $\mathrm{L}$ are typically cylindrical. Channels run through the entire crystal along the cylinder axis and are accessible through $7.1 \AA$ win-

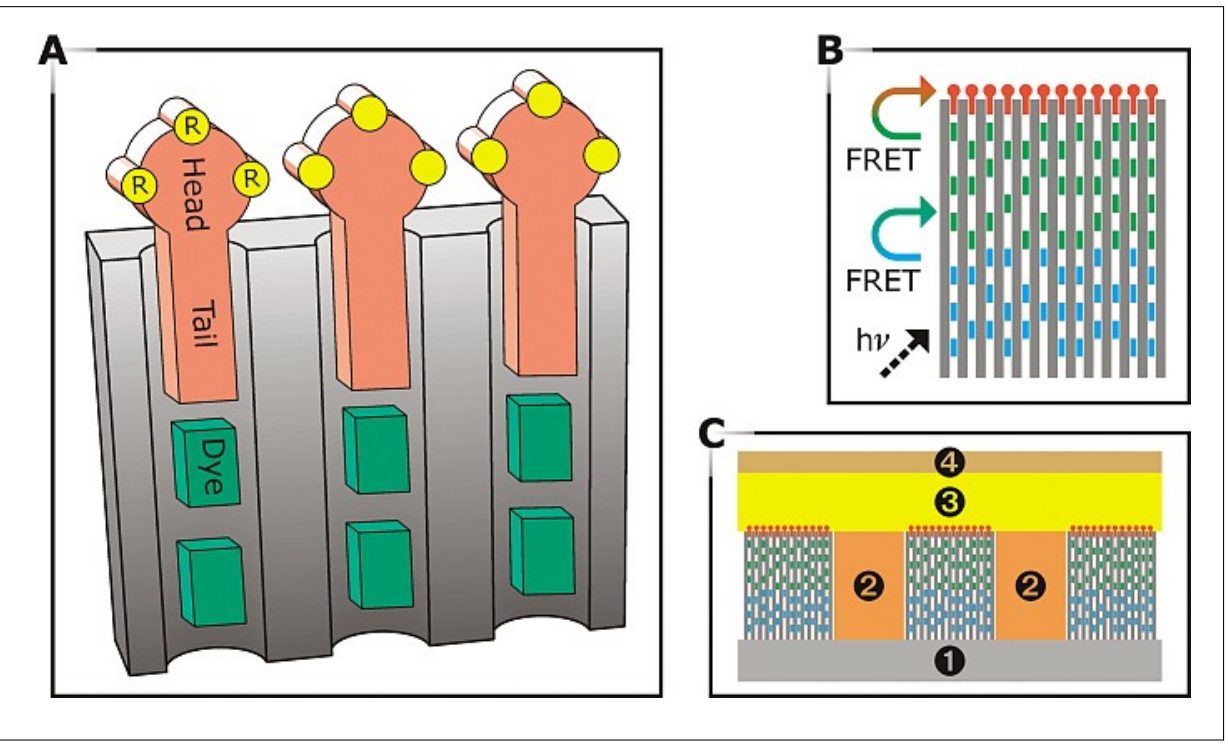

Fig. 1. A) Schematic axial cut through channels containing dye molecules (green) and stopcocks (red) with intrinsic insulating groups (yellow). The channel length depends on the crystal size. B) Nanochannels containing two types of dye molecules (blue and green) and stopcocks (red). Light absorbed by the blue molecules travels to the stopcock heads by FRET via the green molecules. C) Integration of dye-stopcock-zeolite crystals into an organic solar cell (1: contacting oxide, 2: conducting polymer, 3: active material, 4: back contact). 


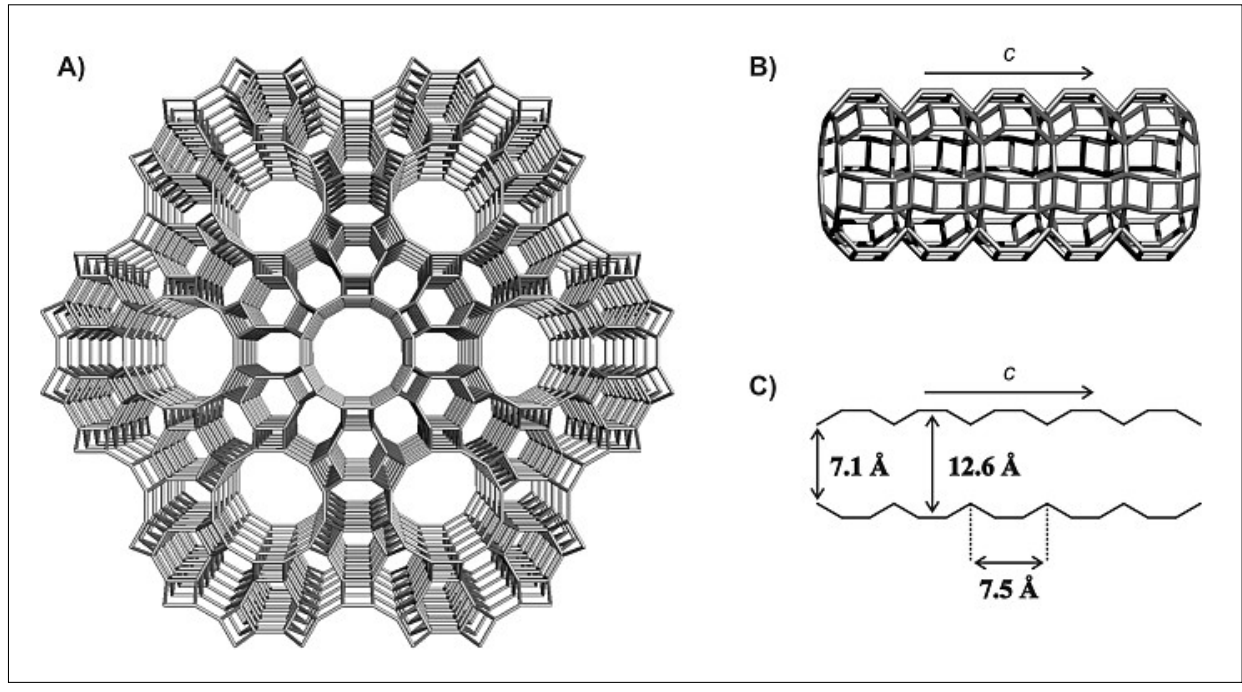

Fig. 2. A) Zeolite $L$ framework projected along the channel axis (c-axis). The lines represent oxygen bridges between $\mathrm{Si}$ and $\mathrm{Si} / \mathrm{Al}$. B) Side view of the main channel and C) its dimensions.

dows located at the base surfaces (see Fig. 2). A zeolite $\mathrm{L}$ crystal of $550 \mathrm{~nm}$ diameter provides approximately 80'000 parallel channels. Different crystal sizes ranging from 30 to $20^{\prime} 000 \mathrm{~nm}$ in length are feasible. ${ }^{[7]}$ The aspect ratio can be tuned to a certain extent, affording disc-shaped or rod-shaped morphologies. A disc-shaped morphology is ideal for the preparation of zeolite L monolayers with the channels oriented perpendicular to the substrate surface. ${ }^{[8]}$ A multitude of cationic and neutral dye molecules has been included into zeolite $\mathrm{L}$ by cation exchange and adsorption from the gas phase, respectively. ${ }^{[1 a]}$ It was generally observed that dye molecules cannot pass each other once they are in the channels. Sequential introduction of different dye molecules therefore results in highly ordered materials featuring dye domains (Fig. 1B). This allows for directed transport of electronic excitation energy along the channels. The energy transfer efficiency of a given donor-acceptor pair thereby depends on the relative orientation of the electronic transition dipole moments and the donor-acceptor distance. The latter should be minimal, but excluding $\pi$-interaction. For the optimization of FRET efficiency, dye molecules with bulky alkyl substituents are ideal. Such moieties are able to inhibit $\pi$-interactions by filling the intrachannel space between adjacent guest molecules.

A mediator is required to extract the electronic excitation energy that has reached the channel entrances via an energy transfer cascade (Fig. 1B). This is accomplished by employing stopcock molecules, which are designed to specifically adsorb at the channel entrances, either by physisorption, electrostatic interaction or covalent bonding. ${ }^{[9]}$ A stopcock molecule contains a tail that can enter the zeolite channel (Fig. 1A). For maximum stability, this tail should carry a reactive group for coupling to amino-functionalized channel entrances. ${ }^{[10]}$ The chromophoric head, which is too large to enter the channels, figures as the acceptor for the electronic excitation energy. Large spectral overlap between donor emission and stopcock head absorption is crucial. Furthermore, parallel alignment of the electronic transition dipole moments of the included donor molecules and the stopcock heads should be provided to ensure efficient FRET. Once the energy has reached the stopcock heads, it is available for transfer to an external component, e.g. the active layer in an organic solar cell. ${ }^{[11]}$ In order to prevent electron transfer, a thin insulating layer should be present between the stopcock heads and the active material. As an elegant solution, we propose to incorporate this insulating layer into the stopcock heads by means of suitable peripheral substituents (Fig. 1A). ${ }^{[12]}$

A possible solar cell design based on oriented monolayers of dye-stopcock-zeolite crystals is shown in Fig. 1C. Charge transport is accomplished by a conducting polymer, such as PEDOT,[13] which is placed between the zeolite crystals. Consequently, the coat of a given cylindrical zeolite crystal is exposed to a different environment than its base. Recent results show that the coat and base surfaces of zeolite L can be functionalized independently, ${ }^{[14]}$ thus allowing for an optimization of these various interfaces. The stopcock molecules are separated from the active material by intrinsic insulating groups, preventing electron transfer (Fig. 1A). Zinc phthalocyanine $(\mathrm{ZnPc})$ is an example of a suitable active material for such a design. ${ }^{[11]}$ $\mathrm{ZnPc}$ features weak or even no absorption between 400 and $550 \mathrm{~nm}$. This gap can easily be covered by a corresponding dye-zeolite antenna.

\section{Luminescent Solar Concentrators}

Aluminescent solar concentrator (LSC) is basically a transparent plate (plastic or glass) containing luminescent centers. Light enters the face of the plate and is partially absorbed and reemitted by these centers. A fraction of the luminescent light is trapped by total internal reflection and guided to the edges of the plate where it can be converted to, for example, electricity by a solar cell. As the edge area of the plate is much smaller than the face area, the LSC operates as a concentrator of light.[15]

The photostability of the dye-matrix composite is an important factor determining the potential of LSCs for photovoltaic energy conversion applications. Direct inclusion of luminescent organic molecules into an organic matrix is particularly problematic in this regard. Introducing suitable dye molecules into nano-sized zeolite crystals and dispersing the resulting dye-zeolite composites in an organic polymer is expected to yield increased photostability. This 'prepackaging' of molecules furthermore opens possibilities for the use of dyes which would be incompatible with a given organic matrix. A first step into this direction has recently been accomplished by the successful preparation of transparent zeolite-polymer hybrids. ${ }^{[16]}$

For strongly luminescent materials there is a considerable overlap between absorption and emission spectra. Self-absorption therefore becomes an important loss mechanism in LSCs. A solution to this problem is the use of a two-dye-zeolite system. Absorption and emission spectra are separated by employing an absorbing species $\mathbf{A}$ and an emitting species $\mathbf{B}$. The concentration of $\mathbf{A}$ is high to ensure efficient light absorption. The electronic excitation energy is transferred along the zeolite channels by FRET to $\mathbf{B}$, which then emits light in a wavelength range where $\mathbf{A}$ does not absorb. As the concentration of $\mathbf{B}$ does not necessarily need to be high, selfabsorption of light emitted by $\mathbf{B}$ becomes negligible. Zeolites containing more than two dyes can be used to create an energy transfer cascade ${ }^{[5 c]}$ and to adjust the absorption properties of the LSC to the spectrum of the incident radiation.

The design shown in Fig. 3 goes a step further by employing oriented dye-zeolite monolayers instead of homogeneously dispersed and randomly oriented dye-zeolite nanoparticles. Such a design is promising in terms of exploiting the defined orientation of electronic transition dipole moments present in dye-zeolite composites. For a given guest molecule in zeolite L, the possible orientations of its electronic transition dipole moment can be described by a double cone-shaped distribution. ${ }^{[17]}$ The opening angle of this cone depends 
to a large extent on the size of the guest molecule. ${ }^{[3]}$ For most of the organic dyes that have been included into zeolite $\mathrm{L}$, the electronic transition dipole moment of the first $\pi * \leftarrow \pi$ transition coincides with the long axis of the molecule. [1a] As a long molecule tends to align with its long axis parallel to the channel axis, the opening angle of the corresponding transition dipole moment double cone is small. Short molecules, on the other hand, typically feature larger opening angles.

Light shining on the face of a LSC plate will be mostly absorbed by dipoles which are oriented parallel to the face. However, such dipoles also have a higher probability of emitting light into the escape cone. The half apex angle of the escape cone is given by $\arcsin (1 / n)$, where $n$ is the index of refraction of the plate. Light emitted into the escape cone will not be totally internally reflected and therefore literally escape (if no reabsorption occurs). An arrangement as shown in Fig. 3 could be used to minimize emission into the escape cone. In a first step, light entering the face of the plate is absorbed by molecules with a wide double cone distribution of electronic transition dipole moments (blue). In a second step, the electronic excitation energy is transported by FRET to an acceptor featuring a narrower double cone distribution (green), therefore having a lower probability of emitting into the escape cone (red). Whether such an arrangement would be beneficial for the efficiency of LSCs has yet to be experimentally verified.

\section{Conclusions}

When using zeolites as host materials, the options for combining different guest species and engineering the photophysical properties are manifold. One-dimensional channel systems, as present in zeolite L, allow for a high degree of supramolecular organization, which can be exploited to obtain energy transfer cascades and orientation of electronic transition dipole moments. The excellent compatibility of zeolites with many different environments and the extensive tunability of dye-zeolite inclusion compounds offer new challenges for implementation in photonic devices for solar energy conversion.

\section{Acknowledgment}

Our research on this topic is financially supported by the European Commission

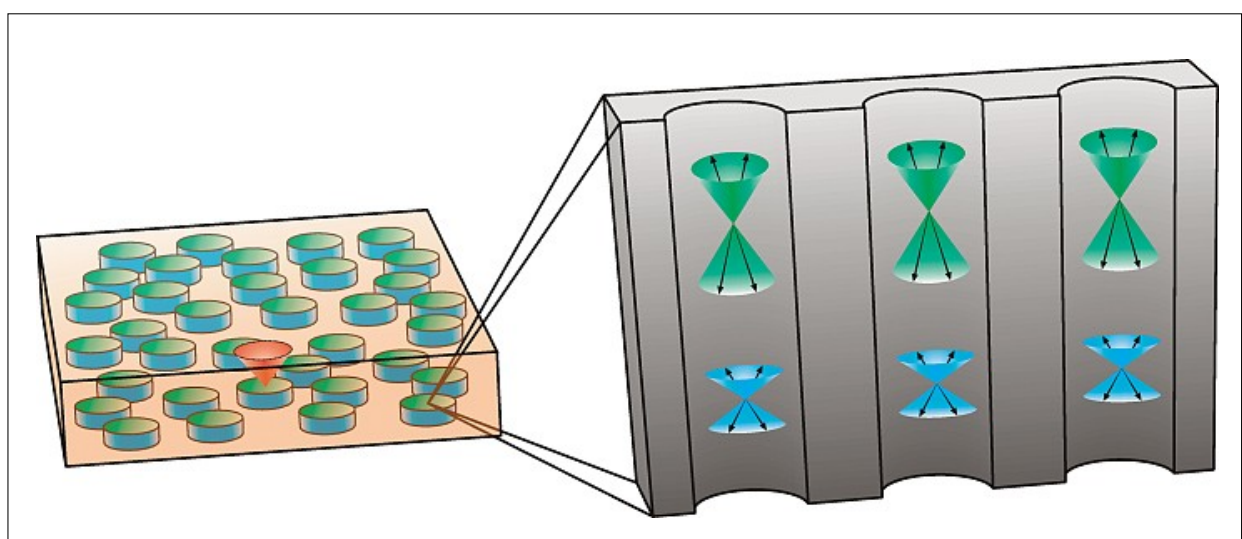

Fig. 3. Luminescent solar concentrator (LSC) based on oriented dye-zeolite crystals. The channels of the disc-shaped zeolite crystals run perpendicular to the face of the LSC. The schematic axial cut (right) shows the double cone distribution of the electronic transition dipole moments for two different dyes. The blue double cones represent the light absorbing molecules (large cone angle), whereas the green double cones indicate the light emitting molecules (small cone angle). The concentration of the latter is kept low to avoid self-absorption. The red cone illustrates an escape cone originating at a light emitting molecule located close to a channel entrance.

through the Human Potential Programme (Marie-Curie RTN Nanomatch, Grant No. MRTN-CT-2006-035884) and by the Clariant Ltd. Research Project 'Dye-Loaded Zeolites'.

Received: October 12, 2007

[1] a) D. Brühwiler, G. Calzaferri, Microporous Mesoporous Mater. 2004, 72, 1; b) G. Schulz-Ekloff, D. Wöhrle, B. van Duffel, R. A. Schoonheydt, Microporous Mesoporous Mater. 2002, 51, 91.

[2] C. Baerlocher, L. B. McCusker, D. H. Olson, 'Atlas of Zeolite Framework Types', 6th ed., Elsevier, Amsterdam, 2007.

[3] H. Maas, A. Khatyr, G. Calzaferri, Microporous Mesoporous Mater. 2003, 65, 233.

[4] C. Minkowski, G. Calzaferri, Angew. Chem., Int. Ed. 2005, 44, 5325.

[5] a) M. L. Cano, V. Fornés, H. Garcia, M. A. Miranda, J. Pérez-Prieto, J. Chem. Soc., Chem. Commun. 1995, 2477; b) H. Garcia, S. Garcia, J. Pérez-Prieto, J. C. Scaiano, J. Phys. Chem. 1996, 100, 18158; c) M. Pauchard, A. Devaux, G. Calzaferri, Chem. Eur. J. 2000, 6, 3456.

[6] a) S. H. Tolbert, A. Firouzi, G. D. Stucky, B. F. Chmelka, Science 1997, 278, 264; b) B. Platschek, N. Petkov, T. Bein, Angew. Chem., Int. Ed. 2006, 45, 1134; c) C.-W. Wu, T. Ohsuna, M. Kuwabara, K. Kuroda, J. Am. Chem. Soc. 2006, 128, 4544.

[7] a) A. Zabala Ruiz, D. Brühwiler, T. Ban, G. Calzaferri, Monatsh. Chem. 2005, 136 , 77; b) Y.-J. Lee, J. S. Lee, K. B. Yoon, Microporous Mesoporous Mater. 2005. 80, 237; c) T. Ban, H. Saito, M. Naito, Y. Ohya, Y. Takahashi, J. Porous Mater. 2007, 14, 119; d) A. Zabala Ruiz, D. Brühwiler, L.-Q. Dieu, G. Calzaferri, in 'Materials
Syntheses', Ed. U. Schubert, N. Hüsing, R. Laine, Springer, Wien, 2008, in press.

[8] a) J. S. Lee, H. Lim, K. Ha, H. Cheong, K. B. Yoon, Angew. Chem., Int. Ed. 2006, 45, 5288; b) A. Zabala Ruiz, H. Li, G. Calzaferri, Angew. Chem., Int. Ed. 2006, 45, 5282.

[9] A. Devaux, K. Lutkouskaya, G. Calzaferri, L.-Q. Dieu, D. Brühwiler, L. De Cola, T. Torres, Chimia 2007, 61, 626.

[10] S. Huber, G. Calzaferri, Angew. Chem., Int. Ed. 2004, 43, 6738.

[11] R. Koeppe, O. Bossart, G. Calzaferri, N. S. Sariciftci, Sol. Energy Mater. Sol. Cells 2007, 91, 986.

[12] L.-Q. Dieu, A. Devaux, I. López-Duarte, M. V. Martinez-Diaz, D. Brühwiler, G. Calzaferri, T. Torres, submitted.

[13] L. Groenendaal, F. Jonas, D. Freitag, H. Pielartzik, J. R. Reynolds, Adv. Mater. 2000, 12, 481 .

[14] a) D. Brühwiler, G. Calzaferri, C. $R$. Chimie 2005, 8, 391; b) M. Busby, H. Kerschbaumer, G. Calzaferri, L. De Cola, Adv. Mater, in press.

[15] a) W. H. Weber, J. Lambe, Appl. Opt. 1976, 15, 2299; b) A. Goetzberger, W. Greubel, Appl. Phys. 1977, 14, 123; c) J. S. Batchelder, A. H. Zewail, T. Cole, Appl. Opt. 1979, 18, 3090; d) J. S. Batchelder, A. H. Zewail, T. Cole, Appl. Opt. 1981, 20, 3733; e) P. Kittidachachan, L. Danos, T. J. J. Meyer, N. Alderman, T. Markvart, Chimia, 2007, 61, 780.

[16] S. Suárez, A. Devaux, J. Bañuelos, O. Bossart, A. Kunzmann, G. Calzaferri, Adv. Funct. Mater. 2007, 17, 2298.

[17] S. Megelski, A. Lieb, M. Pauchard, A. Drechsler, S. Glaus, C. Debus, A. J. Meixner, G. Calzaferri, J. Phys. Chem. B 2001, 105, 25. 